\title{
Research on the Teachers' Effective Guidance to Junior School Students' English Learning on the Basis of Bandura's Social Learning Theory
}

\author{
JIA Xiaoyi*, DING Fangsheng, JI Chunyi, ZHU Yunsi, SHAO Haocong, YAO Shengxia, WANG \\ Jingxing \\ Zhejiang Ocean University, Haida South Road NO.1, Zhoushan City, Zhejiang Province, China \\ *Corresponding Author: JIA Xiaoyi, Zhejiang Ocean University, Haida South Road NO.1, Zhoushan \\ City, Zhejiang Province, China
}

\begin{abstract}
Albert Bandura, the well-known contemporary American psychologist, based on previous studies and studied children's social learning behavior, in 1952 put forward the social learning theory, including the Observational Learning Theory, Interactive Determinism, Self-efficacy Theory and Self-regulated Theory, and its use in cognitive, behavioral and environmental determinants of the interaction to explore people's behavior. Based on the theory of socialism, this paper analyzes the current situation and predicament of junior middle school students' English learning in Donghai Community of Shengsi County, Zhoushan City, Zhejiang Province, and discusses the guiding significance of the theory for English teaching and the enlightenment for improving the initiative and effectiveness of middle school students' English learning
\end{abstract}

Keywords: Social Learning Theory; Junior Middle School English Teaching; Guided Teaching; Independent Study

\section{INTRODUCTION}

Albert Bandura is a famous American psychologist, is also the founder of the modern social learning theory. The innovation of social learning theory proposed by Bandura lies in its breaking through the theoretical framework of traditional behaviorism and focuses more on observing the role of learning and self-regulation in inducing human behavior. Meanwhile, it also attaches importance to the interaction between human behavior and environment. Thereinto, the theory of social learning, which advocates the influence of individual factors and environmental factors on individual behavior, is jointly formed by the theory of the observational learning theory, the observational learning theory, Interactive determinism, self-efficacy theory and self-regulated, self-efficacy and self-regulated. According to the investigation, at present, the research of social learning theory focuses more on the theoretical research, and the practice of a small number of studies have also less involved in high school and junior high school. As middle school is a critical period for English learning of students, how to lay a good foundation for students' English learning in the future and make them find a suitable language learning method at the same time is particularly important.

\section{Problem Analysis}

At the present stage in our country, due to the influence of traditional education mode, some teachers have a deviation in their understanding of teaching methods and models, pursuing curriculum progress and academic performance too much and ignoring the initiative and effectiveness of students' learning. Without the use of a complete set of innovative teaching system, they cannot make good use of existing resources to change the classroom teaching mode. All contents adopt the same teaching method, and they ignore the students' psychological state and the cultivation of students' mental strength. Students fail to form the habit of independent study and the low efficiency of teaching and forced learning finally makes the teacher's teaching achievement and the student's study efficiency are unsatisfactory. The current situation of junior high school students' English autonomous learning ability and the analysis of junior high school English teaching problems have become an important research field of English learning strategies for many Chinese scholars in the context of globalization. 
According to the author's research on the Assessment Questionnaire for Junior High School Students' English Learning Strategies》〉 and Questionnaire for Junior High School Students' English Learning Strategy Awareness and Strategy Emotion compiled by Niu Zhihui, we found that the overall situation of junior high school students' English learning strategies is unsatisfactory. They are highly dependent, and the quality and efficiency of English learning need to be improved. According to the teaching examples and literature at home and abroad, and combined with the survey results, the problems can be divided into 4 aspects:

- Teachers rely on textbooks too much, and cannot deal with teaching problems with flexibility. Besides, teachers cannot implement the new curriculum concept in classroom teaching, namely, to highlight the cultivation of students' language skills, language knowledge, emotional attitude, learning strategies, cultural awareness and other qualities in classroom teaching, leading to students' misunderstanding of their own learning methods.

- The teacher's teaching design lacks originality, with unitary teaching method. And teachers are unable or unwilling to make use of existing resources to assist teaching, which makes teaching lack of context and interest. Moreover, teachers explain more grammar unilaterally, whereas students have fewer oral practice opportunities. Therefore, the classroom atmosphere becomes dull, and students would be in low- learning mood, leading to students' forced learning English.

- In the process of teaching, teachers ignore the cultivation of students' mental strength, failing to effectively guide students to carry out independent learning, and students cannot recognize their own way of English learning better and earlier, so that they can only follow the steps of teachers, and cannot find problems early.

- The rigid teaching form of teachers makes it difficult to provide students with a new way to learn the language, making students blindly rely on classroom learning but ignore independent learning after class, which leads to the difficulty in achieving the expected learning efficiency.

Based on the above problems, we analyzed the current English learning situation of middle school students in specific areas (taking Donghai Community as an example), according to Bandura's social learning theory, we applied psychological theory reasonably and effectively into middle school English teaching, and provided theoretical guidance for changing teachers' traditional teaching methods.

\section{The ConNotation OF Social LEARNing THEORY}

Social learning theory was put forward by J. Dollard and N. Miller around the 1950s, but these theorists inferred human behavior based on animal behavior research, which has limitations. In 1960s, Bandura put forward the modern social learning theory on the basis of the previous research, which broke through the prejudice of traditional behaviorism learning and looked at social learning from the basis of the joint effect of cognition and behavior. [1] Social learning theory is divided into four theories: observational learning theory, Interactive determinism, self-regulated theory and selfefficacy theory.

\section{MATERIALS AND Methods OF THE INVESTIGATION}

\subsection{Object of Research}

The object of this survey is junior high school students in Donghai community of Shengsi County, Zhoushan City, Zhejiang Province. Donghai community is close to residential areas, with a relatively large population (1,769 households, 4,494 permanent residents and 1,300 temporary residents), which is a typical old urban area. Teachers' teaching methods and students' learning habits tend to be conservative and typical. In terms of both teaching and learning methods, there are similar problems with most areas of China, which are suitable for investigation as typical examples. Through teaching, visiting, questionnaire and other forms, we systematically analyzed and understood the learning situation, learning motivation and learning strategies of local students.

\subsection{Overview of Research}

The questionnaire of this research consists of 20 items, which investigate the foreign language learning motivation, language learning difficulty, foreign language learning strategies and other 
factors of the respondents, which can be divided into two aspects: internal factors that affect English learning (learning mentality, learning blind spots and misunderstandings) and external factors (teachers' teaching methods, family factors). Before filling in the questionnaire, we explained the specific requirements for students to fill in the questionnaire. Students could make choices according to their actual situation and submit the questionnaire within the same time. The total number of students participating in the questionnaire survey was 96 , and 96 effective questionnaires were collected.

\subsection{Results and Discussion}

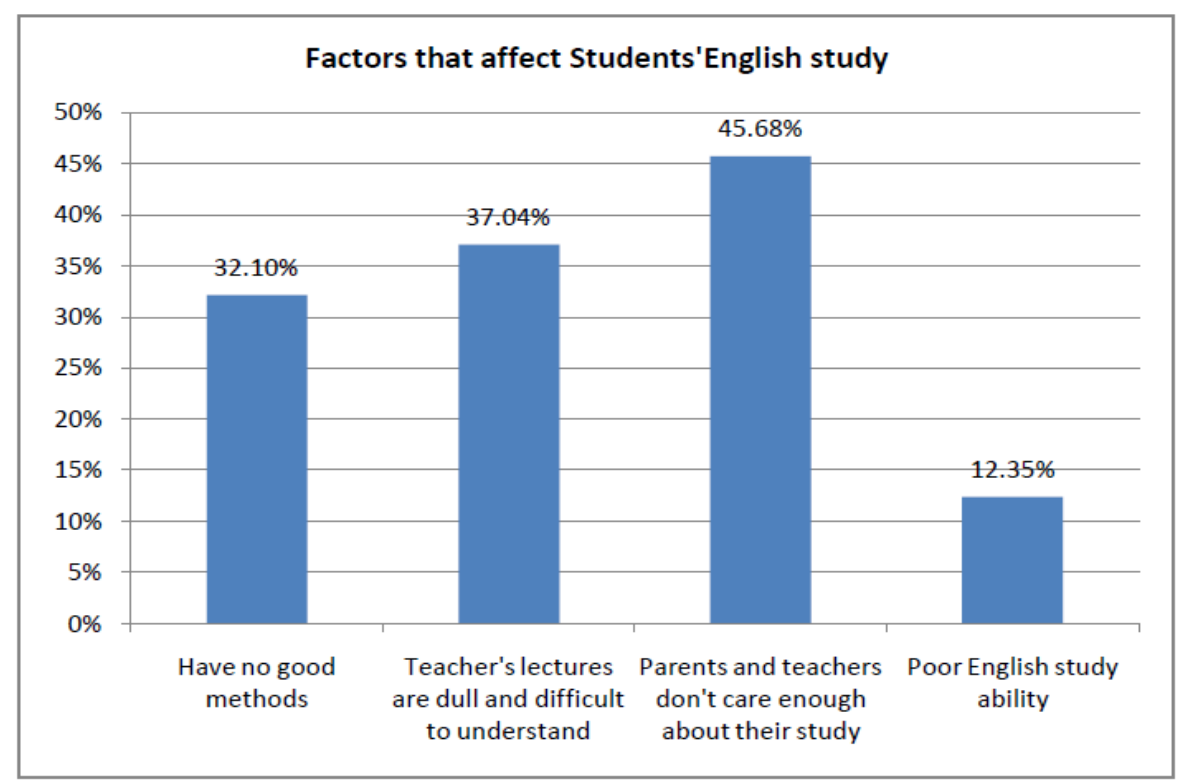

(a)

As can be seen from the figure above, the influence of family and school factors on academic performance accounts for a large proportion, which indirectly reflects the current situation of students' poor learning autonomy, and teachers' correct guidance is urgently needed. "Teacher's lectures are dull and difficult to understand" and "Have no good methods" are also followed. These two points can be seen that the teaching methods still have some omissions and impropriety, which cannot provide students with new perspectives and new ideas for English learning. Teaching is not only boring and rigid, but also makes students develop a dependency psychology. Students only rely on teachers to teach in class instead of sorting out themselves after class, and they do not know how to find their own learning style, which indicates that the operability of English teaching at the present stage still needs to be improved, and the effectiveness of students' learning needs to be paid attention to.

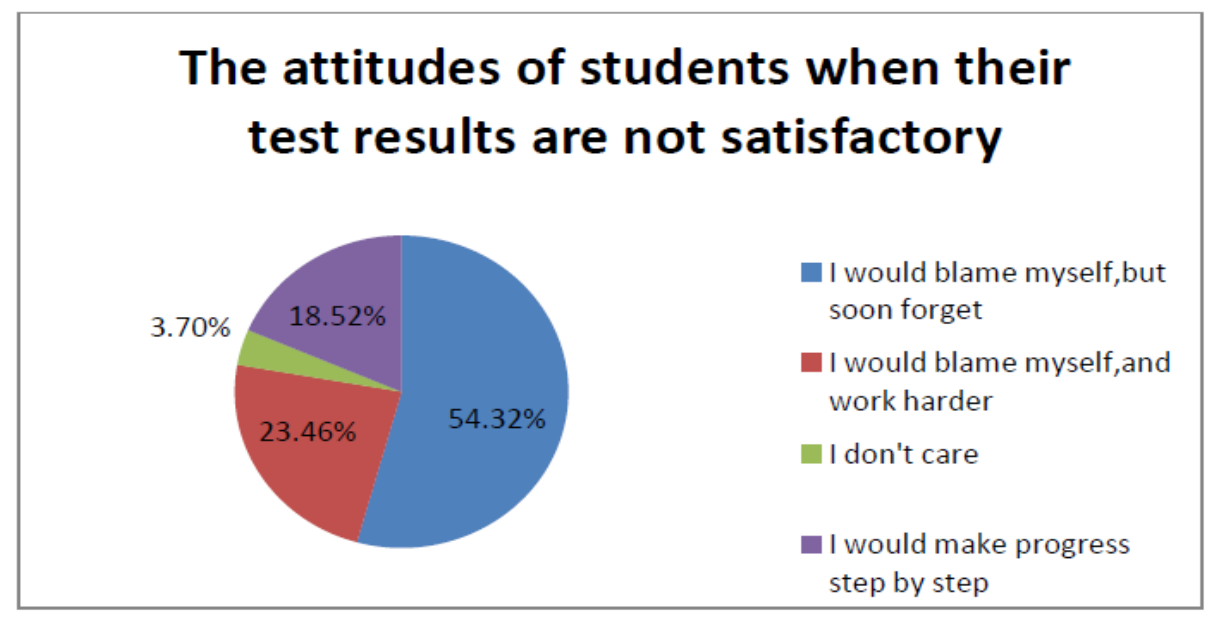

(b)

According to the data above, we find that $77.78 \%$ of students feel self-reproach for their unsatisfactory English performance, but only $23.46 \%$ of students actively adjust their attitude and 
study harder. From this, we can reflect that when students find that their grades do not reach the expected results, most of their responses are forgotten soon. This shows that in the teaching process, teachers guide and actively help students adjust their mentality, which is exactly the key to students' self-active learning desire.

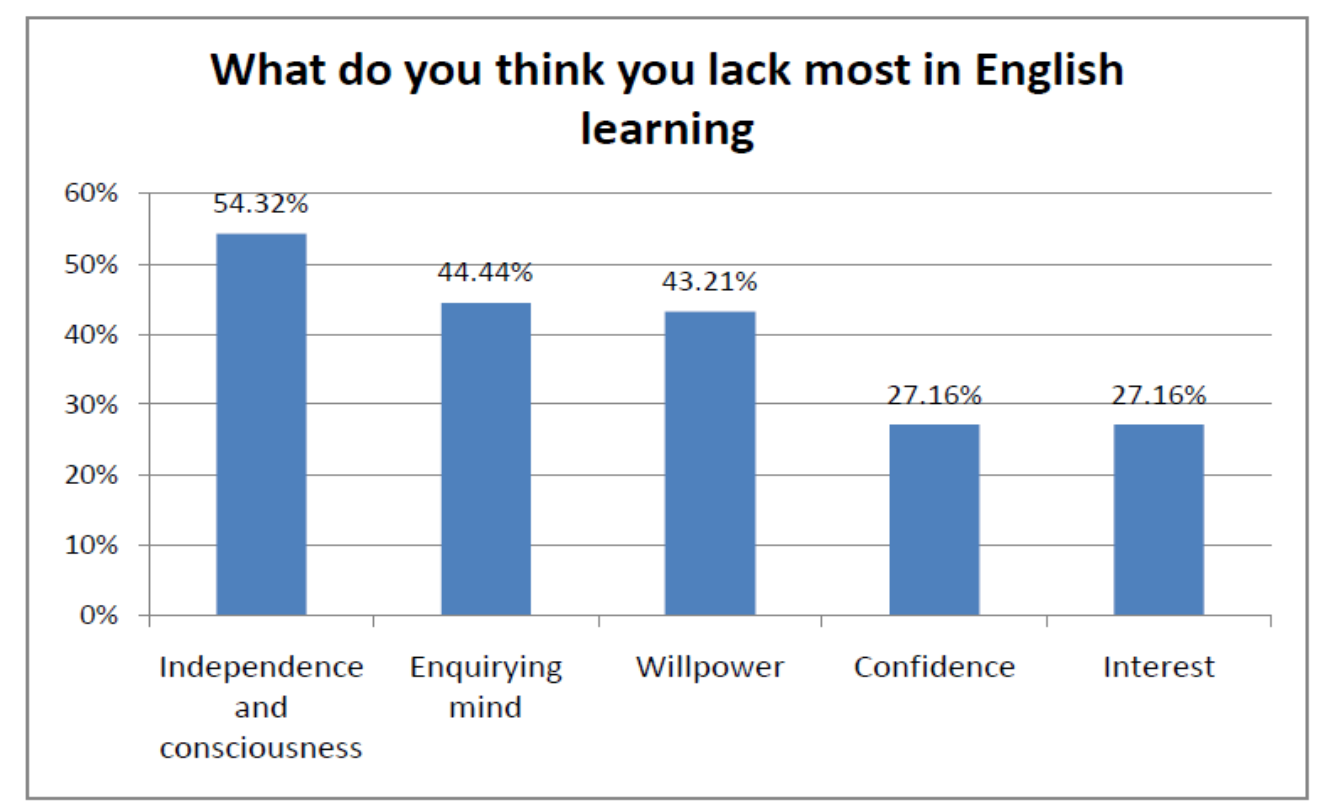

(c)

As shown in figure (c), $54.32 \%$ of the students think that they lack independence and autonomy in study, $44.44 \%$ of the students think that they lack the spirit of research, and $43.21 \%$ of the students think that their willpower is not firm, and a small number of students lack confidence, but these seem to be the most important qualities for a student. Although the real significance of learning and teaching lies in the cultivation of students' self-ability and the display of their potential, teachers' guidance on students' active learning in the actual teaching process is far from enough.

\section{THE APPLiCATION OF SOCIAL LEARNING THEORY}

\subsection{Using Observation Learning Theory to Guide Students to Play the Role of Model}

Social learning is a socialization process in which the society guides members to act in a socially acceptable way, and it is also a learning activity to adapt to the society after observing the behavior of others.[2] The theory of observational learning, as its name implies, refers to the process of people's observational learning, through the observation of the demonstrative activity, so as to receive and guide the generated imitation action. For students who have first contact with foreign languages, it is difficult to find a suitable learning method at the very beginning. Therefore, it is effective to improve students' learning effect to get inspiration through observation.

Bandura argues that people's behavior is largely learned through the observation of role models (indirect experience), except through direct experience.[3] Combined with the survey results in figure (a) and according to the connotation of the theory of observational learning, we can conclude that the method of influencing students' learning behavior through observing others is of great benefit to middle school students' English learning. In the process of learning, students are not only influenced by the learning status of the students around them, but also subtly observe and absorb the learning style of the students around them, hoping to improve their learning efficiency and performance, so as to reach a higher level. According to the observational learning theory, if the demonstrative behavior repeatedly shows the expected response of learners, the learner will be subconsciously influenced to reproduce the behavior, giving guidance when failure and encouragement when success, then the demonstrative behavior will be effective in most people. Teachers should timely observe and understand the learning situation of their students, looking for and summarizing the learning methods of excellent students, so as to set an example for other students and design a suitable learning model for students who cannot find a good way. It is reasonable and effective to guide students to learn how to observe learning by taking the four processes of observation learning theory as basic, such as 
attention, maintenance, generation and motivation.

\subsection{Using Interactive Determinism to Create a Vivid Language Learning Environment and Guide Students to Try Various Learning Methods}

Interactive determinism emphasizes the interaction among behavior, cognition and environment in social learning. [4] Bandura believes that human behavior potential and environment are a two-way interaction, and do not work independently. English is different from other subjects. As a language, it emphasizes more on the influence of language environment. For students who root in China and study foreign languages at home, they have never had or had little contact with English before, and it is very difficult or even painful for them to learn this strange or even completely unfamiliar language for the first time without a natural language environment. If we can provide students with an atmosphere of learning language environment, it will stimulate their learning potential. Similarly, once students' learning potential and motivation are stimulated, they will give a positive impetus to the overall environment, which in turn will affect the individual students. This provides a difficult problem for the current domestic teachers: how to use existing resources to create a language environment suitable for students to learn English, so that students can use it more freely and fluently and improve learning efficiency, which is a problem that needs to be studied in contemporary teaching.

The relationship among the subjects, behavior and environment is determined by two and two mutually, and the relationship among the three is a continuous and dynamic interactive determining relationship. Therefore, in order to perform a certain kind of behavior, people need to have environment in addition to motivation, especially for English teaching.[3] "Teaching equipment and teaching environment affect the improvement of English learning to some extent". It is a common problem in English teaching and learning. In some areas, it is the most important problem. As professor Liu Runqing points out, "Most second language learners study in countries where the place is not their mother tongue. Only a little language is heard in a limited amount of class time, and not for communication."[3] China is different from Europe, America and some southeast Asian countries and regions, the Chinese language environment limits its foreign language learning. Just rely on the teacher's teaching in class, and which is to enhance academic performance as the goal, would greatly reduce the usefulness and interestingness of English learning. All these will lead to the psychological state of "forced" and "helpless" in students' learning, which makes students' learning efficiency and learning consciousness not high. However, English education in middle school is a key period for students to lay a good foundation, which is crucial to their interest in learning English in the future as well as efficiency and methods. Therefore, this puts forward higher requirements for middle school teachers' teaching, and the correct guidance has become the key for students to improve the initiative and effectiveness of English learning.

Nowadays, most middle schools equip their classrooms with multimedia and other teaching equipment. Teachers can use this resource to enrich their teaching methods. There are some examples:

- Let students watch English movies and learn popular English songs regularly;

- Let the students listen to the English radio in the morning and imitate the pronunciation.;

- Organize English debate competitions regularly to make full use of the English knowledge learned in class.

- Regularly subscribe to foreign English periodicals for students;

- On the day of traditional festivals in the west, teachers could explain the origin of festivals and folk culture to the students;

- Invite foreign friends or foreign teachers to provide guidance.

- Teachers should encourage students to watch the European and American popular TV dramas after class(Such as Young Shelton, Modern Family and The Simpsons, etc), which is given priority to with English subtitles, native language subtitles are complementary way, and students can excerpt unfamiliar words and phrases in time. If they can persist for a long time, they would build an environment to learn English for themselves.( According to the author's personal experience and survey results, students with the habit of watching American TV series and British TV series 
generally have better English scores than those who only do exercises and memorize words. Moreover, students are more interested in learning English, and they are more flexible, openminded and have a global perspective.)

- Encourage students to read the European and American pop novels(Such as Harry Potter, The Bookworm Series, etc.) Looking for interesting books that attract teenagers, so that students can have a deeper understanding of the use of language and the customs and cultures of other countries, therefore, they can have a spontaneous interest in learning English instead of the "Chinglish" in textbooks.

English teachers should actively guide students to create a language environment for learning a foreign language by students themselves, so that they can love this new language from the bottom of their hearts and develop the habit of independent learning, hence they will no longer "hit" the classroom content and learn rigidly. When most students are influenced by this learning atmosphere, the remaining few students will be driven to form a good atmosphere for learning English, so as to promote the improvement of students' academic performance.

\subsection{Using Self-Regulated Theory to Guide and Help Students to Learn to Make Self- Observation, Self-Judgment and Self-Evaluation}

Bandura believes that self-regulation is an internal reinforcement process of individuals, a process in which individuals regulate their behaviors by comparing and evaluating their plans and expectations for behaviors with the actual results of behaviors. [4]Bandura's self-regulation theory focuses on the self-regulation of learners, and students make self-evaluation according to individual standards, social comparison, group comparison and other judgment standards. The final effect of students' learning depends largely on their mentality, especially for middle school students who are still in adolescence, their mood and mentality are relatively floating, and they are in the period of personality shaping. Learning how to correct self-regulation and attitude, and make a right self-assessment and feedback can help students cope with various problems in life and study better. Teachers need to use this theory to help and guide students, and regulate their emotions and adjust their learning attitude timely.

In the learning period, students should learn not only knowledge, but also the ability of selfregulation, which is the key to enable students to produce independent learning ability. We ought to teach students to effectively self-regulate and self-manage their own learning or activities, which should become the direction of our teaching reform.[5] In the process of English teaching, especially in different learning stages, teachers need to grasp the specific content of the theory in order to guide students better and effectively.

This process is divided into three stages:

- First of all, students should set up systematic learning objectives and contents according to selfregulated learning objectives, then arrange learning plans reasonably, and estimate the results of learning.

- Secondly, let the students to study as planned, during the process, they can learn to make a selfobservation, and regular self-reflection, so as to correct and adjust their learning attitude.

- At the end of the learning tasks at each stage, students should make timely feedback and objective and fair evaluation of the learning results, that is, self-evaluation, and make corresponding learning schedule and status adjustment, so as to face the next stage of learning with a better attitude.

In the long learning process, at the same time through the cultivation of self-regulation ability, students will have more confidence in learning, not only correct the state of mind, but also develop a set of their own learning methods.

\subsection{Using the Theory of Self-efficacy to Pay Attention to the Cultivation of Students' Mental Strength and Guide Them to Form a Right Self-consciousness}

Self-efficacy refers to an individual's judgment, belief or subjective self-assurance and feelings about whether he can complete an activity at a certain level. "Performance expectations affect not only the 
choice of event and venue, but also the level of effort," Bandura pointed out.[4] Different from selfregulation theory, efficacy theory emphasizes more on the influence of people's expected attitude.

Self-efficacy is a part of the central force of pedagogical theory. "Mental force refers to the individual's non-cognitive psychological ability, including emotional motivation, willpower, attention, self-evaluation and regulation."[6] It can be seen that the cultivation of mental energy is one of the most important aspects in the teaching process. It not only cultivates students' learning ability, exercises their autonomy, but also shapes their firm characters. In the whole process of students' independent learning, the cultivation of mental effort runs through. Only the students who have a certain ability of mental strength can plow out their own struggle journey of learning.

Because junior high school students are in the stage of puberty, beginning to appear adolescent psychological germinating, independent self-consciousness enhancement and other psychological changes, therefore, there are more requirements for students to cultivate in this period of time (Such as emotional power, willpower, attention, self-evaluation, control and other mental capacity). In view of this, Junior high school teachers should apply self-efficacy theory to teaching practice, not only attach importance to the psychological development of students, and understand the student's psychological demands, but also pay attention on every phase of the students' study and give different guidance to different students. When students succeed, they should be affirmed, and when they fail, they should be encouraged and guided. In the long run, students' mental strength will be gradually cultivated. Their goals will be firmed with confidence, and they could also form a correct sense of self at the beginning of learning. Students will not be conceited in victory or depressed in defeat, and they will improve their independence and autonomy in English learning.

\section{CONClusions}

On the basis of the previous literature and practical survey data, this paper demonstrates and integrates the guiding significance of Bandura's social learning theory to middle school English teaching. As a language, English learning is very different from other subjects, not only relying on "rote learning", English teachers need to pay attention to this difference and give flexible teaching. Unfortunately, many teachers think that this kind of teaching model will slow down the teaching progress and thus neglect to cultivate students' interest in western culture. They don't know that if students can have a deep understanding of other countries' culture and thinking mode, and be familiar with the cultural difference between their own country and other countries, they can have a better understanding of this language, not only mastering the learning rules of this language, but also developing interests in English learning, finally, they would improve their autonomy pf learning. This method not only needs to be integrated into the teacher's teaching in class, but also requires the teacher to teach this kind of learning method to students and guide them to try.

In the actual teaching process, in addition to the change of teaching methods, teachers should pay more attention to the importance of guiding students to learn independently. At the beginning of students' contact with this language, it is necessary to attach importance to students' learning psychology, but not to make students weary of learning. Moreover, teachers should also help their students to overcome the difficulty in time, and let them find their own way of English study gradually in the process. The change of the role of teacher from "imparting" to "leading" will lead students to gradually learn to face and deal with the problems they encounter in foreign language learning independently and confidently, so as to improve the initiative and effectiveness of learning.

\section{ACKNOWLEDGEMENTS}

First of all, I would like to extend my sincere gratitude to my professor, Ding Fangsheng, for her instructive advice and useful suggestions on my thesis. I am deeply grateful of her help in the completion of this thesis.

High tribute shall be paid to the co-authors and other people who worked for it.

Finally, I am indebted to the sponsor of this paper: National Innovation and Entrepreneurship Training Program for College Students. 


\section{REFERENCES}

[1] Bandura. Social Learning Theory. Publisher: China Renmin University Press, China, 2015(1); PP.10-11.

[2] Jiangxiao. Bandura with the Social Learning Theory. Publisher: Social Sciences, 1987.(1)

[3] Zhu Cuihua. Bandura's Social Learning Theory and Oral English Teaching in College. Publisher: Modern Educational Management, 2009.(9)

[4] Ren Zhaoxia, Chen Ping. Bandura's Social Learning Theory and its Application in Education. Publisher: Journal of Shandong Agricultural Management Cadre Institute, 2004.(5)

[5] Xu Zhonggeng. Self-regulated learning theory and its teaching significance. Publisher: East China Normal University, 2001.(10)

[6] Rong Zhongkui. On the Practical Educational Significance of Bandura's Social Learning Theory. Publisher: Higher Education Forum, 2002.(12)

\section{AUTHOR BIOGRAPHY}

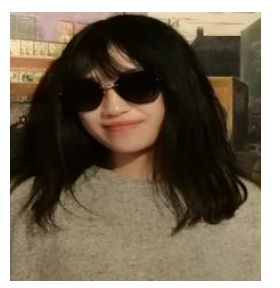

JIA Xiaoyi, born in Shanxi Province, China, a junior English major student of Zhejiang Ocean University, focuses on English language studies.

Citation: JIA Xiaoyi, DING Fangsheng, et.al. "Research on the Teachers' Effective Guidance to Junior School Students' English Learning on the Basis of Bandura's Social Learning Theory". International Journal of Humanities Social Sciences and Education (IJHSSE), vol. 6, no.10, 2019, pp. 72-79. doi: http://dx.doi.org/ 10.20431/2349-0381.0610008.

Copyright: () 2019 Authors. This is an open-access article distributed under the terms of the Creative Commons Attribution License, which permits unrestricted use, distribution, and reproduction in any medium, provided the original author and source are credited. 